\title{
Behavioral Responses of Aphid (Myzus Persicae) to Volatiles from Non-Host Plant Lavender (Lavandula Angustifolia) *
}

\author{
Ting-Ting Cai, Xiang-Qun Lin and Pu-Xian Zhang \\ Yunnan Forestry Technological College \\ Kunming, Yunnan, 650224, China \\ E-mail: ctingting2004@163.com \\ Kun Zhang \\ Southwest Forestry University \\ Kunming, Yunnan, 650224, China \\ Liang Wang \\ Yunnan Tobacco Company Kunming Branch, \\ Kunming, Yunnan, 650051, China \\ Min $\mathrm{Ye} \dagger$ \\ Key Laboratory of the Ministry of Education for Agro-Biodiversity \\ and Pest Management, \\ Yunnan Agricultural University, \\ Kunming, Yunnan, 650201, China \\ $\uparrow$ E-mail address:yeminpc@126.com
}

\begin{abstract}
Volatile organic compounds (VOCs) can have an important influence on plant-insect interactions. In particular, insect behaviors are often influenced by the volatiles of both host plants and non-host plants. The objective of this study was to assess the behavioral responses of the tobacco aphid, Myzus persicae to the volatiles of Lavandula angustifolia (Lamiaceae). Our studies using a range doses of plants have shown that, When the mass of $L$. angustifolia was more than or equal to $4.0 \mathrm{~g}$, volatiles emitted from plants showed repellent effects on $M$. persicae, but at masses of less than $4.0 \mathrm{~g}, M$. persicae did not exhibit significantly preference. These findings provide new insights into the interactions between $L$. angustifolia plants and M. persicae, which will contribute to the development of new management strategies for biological control of insect pests.

Keywords: Behavioral Responses; Aphid; Myzus persicae; Lavandula angustifolia; Y-tube lactometer.

\footnotetext{
* This research was supported by the guidance project of science fund from Education Department of Yunnan Province (Grant No.2016ZDX176) and college scientific research project from Yunnan Forestry Technological College.
} 


\section{Introduction}

Plants naturally not only literally building forests from gases taken from the air but also releasing complex bouquets of volatile organic compounds (VOCs) back into the air, these chemical signatures can be used by predators and parasitoids to locate their prey or hosts[1].Thus, VOCs can have an important influence on plant-insect interactions. For example, host plant volatiles can regulate insect behaviors [2, 3]. The volatiles of maize (Zea mays) are attractive to Western corn rootworm adults (Diacritica virgifera) [4], and the odour of oilseed rape (Brassica napus) attracts cabbage seed weevils (Ceutorhynchus assimilis) [5]. Moreover, non-host plants volatiles also can influence insect behaviors [6]; Cymbopogon citrates, a plant with known repellent activity, have a repellent effect against mosquitos [7], as do volatiles of the neem tree [8]. Thus, intercropping of host plants with non-host plants can be used, so that the VOCs of non-host plants disrupt the ability of pest insects to find host plants. This may be an effective means of biological control of insect pest.

The tobacco aphid, Myzus persicae, is one of the main pests that feed on the leaves of tobacco (Nicotina tabacum), to the extent that it's aggregative behaviors can seriously reduce the yield and quality of tobacco. Although chemical control is effective, overreliance on this method carries health and environmental risks associated with the residues that remain on tobacco leaves, along with the resistance that herbivores may develop which in turn may lead to the resurgence of primary pests and outbreaks of secondary pests [9]. Therefore, it is prudent to explore alternatives to chemical control to improve the sustainability of pest management [10]. Methods that alter herbivore behavior have been recognized as potential alternative tools for the control of insect pest populations [11].

Field observations have indicated that the plant, L. angustifolia, is never damaged by aphids. We hypothesized that the volatile compounds present in $L$. angustifolia, May have repellent effects to aphids. The main objective of this study was to test whether the volatiles of $L$. angustifolia have repellent effects on aphids. Confirmation of such properties would provide a useful basis for the development of intercropping methods with L. angustifolia, in order to reduce damage caused by aphids to tobacco and other plants. 


\section{Materials and Methods}

\subsection{Plants}

L. angustifolia were reared in plastic pots $(11 \mathrm{~cm}$ diameter, $9 \mathrm{~cm}$ height) in a climate-controlled greenhouse room $\left(25^{\circ} \mathrm{C}, 70 \% \mathrm{RH}, \mathrm{L} 16\right.$ : D8) and watered every three days in the morning. No fertilizers were applied.

\subsection{Insects}

The ablate adult aphids ( $M$. persicae) were originally collected from infested tobacco, at the Yunnan Agricultural University, Kunming, China. They were all reared on potted tobacco (at the stage with 5-12 true leaves) in well ventilated plastic cages $(50 \times 50 \times 50 \mathrm{~cm} 3)$ kept in separate greenhouse chambers at $22 \pm$ $2^{\circ} \mathrm{C}, 50 \%-72 \% \mathrm{RH}$, and a photoperiod of 16:8 L:D.

\subsection{Y-tube Olfactometer Bioassay}

Behavioral responses of the aphids to L. angustifolia volatiles were investigated with a glass Y-tube olfactometer $(1 \mathrm{~cm}$ in diameter, $10 \mathrm{~cm}$ stem and $10 \mathrm{~cm}$ arms, set at a $75^{\circ}$ inner angle). Air was pumped through an activated charcoal filter to remove any contaminating odors and a humidifier was used to humidify the air, divided by a glass Y-junction. The two airflows then passed through two separate flow meters, which regulated the flow rate to $133 \mathrm{~mL} / \mathrm{min}$. The air passed into two glass cylinders $\left(11 \times 19 \mathrm{~cm}^{2}\right)$ with quick fit lids, into which the volatile sources were placed. From there, the air from the glass cylinder flowed into the arms of the olfactometer. All tests were conducted in a climate room $\left(25 \pm 1^{\circ} \mathrm{C}, 50-70 \% \mathrm{RH}\right)$ under a $55-\mathrm{W}$ fluorescent lamp that emitted daylight wavelengths, fitted with a prismatic filter to ensure a completely even distribution of light. This illuminated the Y-tube with a light intensity of $865 \mathrm{~lx}$.

For each of the bioassays, 50 adult aphids were tested following established protocols [12]. The aphids were introduced individually into the entry arm. Timing started when the individual reached the beginning line $(3 \mathrm{~cm}$ from the stem inlet), and we then began to observe their choices in the Y-tube Olfactometer. The observation period ended when the individual reached the finish line ( $7 \mathrm{~cm}$ from the $\mathrm{Y}$-junction) of one of the lactometer arms, with maximum observation duration of 5 min per test.

Individuals that did not choose a side within $5 \mathrm{~min}$ were recorded as "no choice". After five insects had been tested, the Y-tube was cleaned with alcohol $(75 \%)$ and distilled water, left to dry, and subsequently the odor sources were switched between the left and right side arms to minimize any spatial effect on 
choices. Each individual was tested only once. Ten different aphids were used for each replication and five replications were carried out for each treatment.

The experiment was conducted, to examine the response of $M$. persicae to odors emitted from L. angustifolia volatiles versus clean air. Five different masses of aerial parts of L. angustifolia plants were established as five treatments respectively, with five replicates were carried out for each treatment. About 1 hour after taking the five different masses of aerial parts, we placed the aerial parts of $L$. angustifolia plants in the glass cylinder respectively, to minimize any mechanical damage to the odor sources.

\subsection{Statistical Analysis}

For the bioassays with Y-tube olfactometer, Chi-square tests were used to test the hypothesis that the distribution of side-arm choices between pairs of volatiles deviated from the null model that volatile sources were chosen with equal frequency. Independent-sample t-tests were used to assess significant differences between the mean time that was required for individual aphids to make a choice between different volatiles. All the data were analyzed with statistical package SPSS 17.0.

\section{Results}

The results from the olfactometer experiment that investigated insect responses to L. angustifolia volatiles versus clean air showed that aphids were significantly repelled by odors emitted from $L$. angustifolia when the mass of plant tissue was more than or equal to $4.0 \mathrm{~g}(\mathrm{p}<0.05)$ (above set doses) (Fig. 1). While the masses was less than $4.0 \mathrm{~g}, M$. persicae did not exhibit significantly preference $(\mathrm{p}>0.05)$.

There was no significant difference in choice time for the odour sources (i.e., L. angustifolia versus blank treatment), except when the mass of L. angustifolia was $1.0 \mathrm{~g}$ (above set doses) ( $\mathrm{p}>0.05)$ (Fig. 2). When the pair of odors included $1.0 \mathrm{~g}$ of L. angustifolia versus the blank treatment, aphids' speed of movement toward the odors of $L$. angustifolia was significantly faster than the blank treatment $(\mathrm{t}=2.21, \mathrm{p}=0.032<0.05)$ (Fig. 2). 


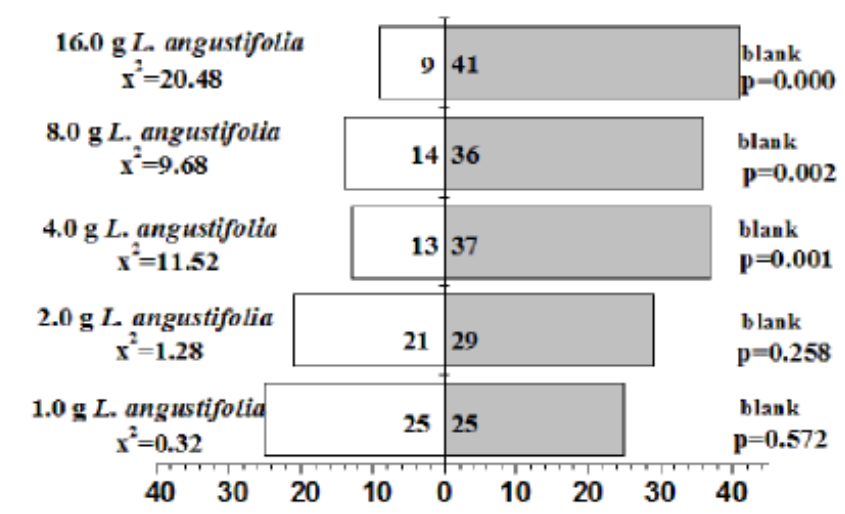

Fig.1. Olfactory response of $M$. persicae to odors from $L$. angustifolia versus blank, "Blank" indicates clean air as the control group. Numbers in bars represent individual aphids that moved toward the volatiles on five different experimental doses.

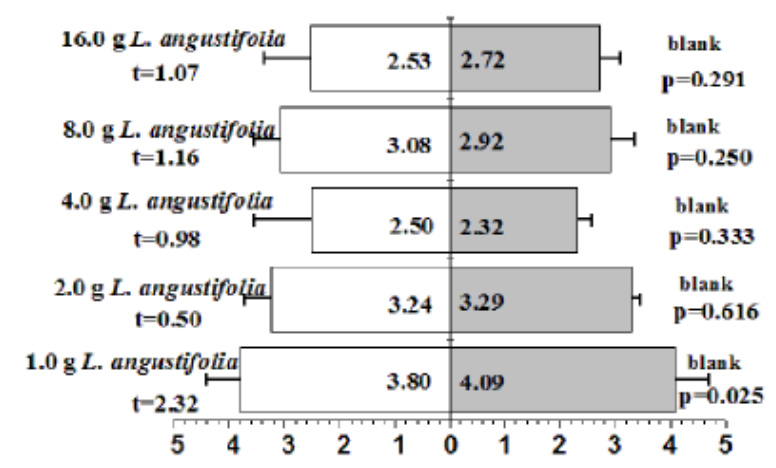

Fig. 2. Olfactory response of $M$. persicae to odors from L. angustifolia versus blank, "Blank" indicates clean air as the control group. Numbers in bars represent the mean time (min) that it took individual aphids to move toward the volatiles.

\section{Discussion}

It is well documented that insect behavior is guided by plant physical cues, such as color and shape, along with short-range chemical cues, such volatile organic compounds of plants, or some secretions produced by insects ${ }^{[13,14]}$. Our study indicates that the behavior of M. persicae was influenced by the volatiles of $L$. angustifolia. M. persicae were significantly repelled by odors emitted from $L$. angustifolia when the mass of plant tissue was more than or equal to $4.0 \mathrm{~g}$. These findings suggest that the $L$. angustifolia volatiles represent a chemical cue that influences the behavior of M. persicae, although L. angustifolia is not their host plant. These volatiles may contain some compounds that repel aphids that feed on tobacco leaves. 
In addition, in another olfactometer study, the odour of volatiles from the host plant tobacco attracted aphids ${ }^{[6]}$, whereas our study indicates that the odour of L. angustifolia volatiles was significantly repellent to $M$. persicae. So, tobacco plants have potential for use in intercropping with L. angustifolia plants, so that the volatiles of $L$. angustifolia plants may disrupt host finding by aphids. In this way, it may be possible to use non-host plants volatiles to mediate the location of aphids. If successful in the field, such a system could be valuable for reducing the need for chemical applications, and ultimately result in economic benefits to farmers.

\section{References}

1. S.B. Vinson, Host selection by insect parasitoids. Annual review of entomology, Vol.21 (1976), pp. 109-133.

2. J. Visser, Host odor perception in phytophagous insects. Annual review of entomology, Vol.31 (1986), pp. 121-144.

3. D.J. Wei, Plant-insect Chemical Communication and Its Behavior Control Acta Photophy siologica Sinica, Vol.27 (2001), pp. 193-200.

4. B. Hibbard, T. Randolph, E. Bernklau, E. Abou-Fakhr and L. Bjostad, Electroantennogram-active components of maize silk for adults of the western corn rootworm (Coleoptera: Chrysomelidae). Environmental Entomology, Vol.26 (1997), pp. 285-296.

5. E. Bartle, M. Blight, A. Hick and I. Williams, The responses of the cabbage seed weevil (Ceutorhynchus assimilis) to the dour of oilseed rape (Brassica napus) and to some volatile isothiocyanates. Entomologia experimentalis et applicata, Vol.68 (1993), pp. 295-302.

6. S.F. Nottingham, J. Hardie, G.W. Dawson, A.J. Hick, J.A. Pickett, L.J. Wadhams and C.M Woodcock, Behavioral and electrophysiological responses of aphids to host and nonhost plant volatiles. Journal of chemical ecology, Vol.17 (1991), pp. 1231-1242.

7. W.S. Leal and K. Uchida, Application of GC-EAD to the Determination of Mosquito Repellents Derived from a Plant, Cymbopogon citratus. Journal of Asia-Pacific Entomology, Vol.1 (1998), pp. 217-221.

8. M.F. Balandrin, S.M. Lee and J.A. Klocke, Biologically active volatile organosulfur compounds from seeds of the neem tree, Azadirachta indica (Meliaceae). Journal of agricultural and food chemistry, Vol.36 (1988), pp. 1048-1054.

9. A. Hart, C.D. Brown, K.A. Lewis and J. Tzilivakis, p-EMA (II): evaluating ecological risks of pesticides for a farm-level risk assessment system. Agronomies, Vol.23 (2003), pp. 75-84. 
10. L.L. Cui, J. Dong, F. Francis, Y.J. Liu, S. Heuskin, G. Lognay, J.L. Chen, C. Bragard, J.F. Tooker and Y. Liu, E- $\beta$-farnesene synergizes the influence of an insecticide to improve control of cabbage aphids in China. Crop Protection, Vol.35 (2012), pp. 91-96.

11. L.C. Rodriguez, and H.M. Niemeyer, Integrated pest management, semiochemicals and microbial pest-control agents in Latin American agriculture. Crop Protection, Vol.24 (2005), pp. 615-623.

12. Y.G. Lou, B. Ma, and J.A. Cheng, Attraction of the parasitoid Anagrus nilaparvatae to rice volatiles induced by the rice brown planthopper Nilaparvata lugens. Journal of chemical ecology, Vol.31 (2005), pp. 2357-2372.

13. E. Hatano, G. Kunert, J. Michaud and W.W. Weisser, Chemical cues mediating aphid location by natural enemies. European Journal of Entomology, Vol.105 (2008), pp. 797-806.

14. J. Michaud and M. Mackauer, The use of visual cues in host evaluation by aphidiid wasps: I. Comparison between three Aphidius parasitoids of the pea aphid. Entomologia experimentalis et applicata, Vol.70 (1994), pp. 273-283. 